Journal of Engineering and Applied Sciences 14 (Special Issue 1): 3885-3890, 2019

ISSN: 1816-949X

(C) Medwell Journals, 2019

\title{
Stability Analysis of TTR under VIV and Parametric Excitation
}

\author{
Ranjeet Kr Verma and Himanshu Uppal \\ Department of Naval Architecture and Offshore Engineering, AMET University, Chennai, India
}

\begin{abstract}
This study analyzes the non-linear dynamic behaviour and stability of Top Tensioned Riser (TTR) under combined effect of in line vortex formation and parametric excitation due to nonlinear drag term of wave motion. While analyzing the response of TTR general approach is either to neglect effect of vortex formation which is because of current flow by passing the TTR or parametric excitation because of changes in tether tension. However, in real sea conditions coexistence of both loads may produce disastrous chaotic response. In this study, mathematical model of TTR considering pinned-pinned end condition is derived and various design parameters of TTR is explored, considering vortex shedding frequency to be twice of fundamental structural frequency of TTR using Monte-Carlo Simulation.
\end{abstract}

$\underline{\text { Key words: TTR, vortex, parametric, Monte-Carlo Simulation, disastrous, fundamental }}$

\section{INTRODUCTION}

Tension Leg Platform (TLP) is positively buoyant structure which is vertically moored to sea bed by tethers. TLP draws its static stability in vertical plane because of tether pretension. TLP can be used in wide range of water depth from shallow to deep water. Now a days various innovative TLP design is considered as economical solution for foundation of large capacity wind turbines and many offshore oil and gas platform. To increase the payload capacity of TLP, reduction in pretension of tether is required which in turn may cause significant stability issues for tethers and TLP platform.

TLP is a positive buoyant structure, so, it is installed with TTR (Top Tensioned Riser). TTR's are very stiff in axial direction due to positive buoyancy and fluctuation in axial tension, sometime causes significant coupling of the system (Chen et al., 2012; Josefsson and Dalton, 2010; Sanaati and Kato, 2012; Wilde and Huijsmans, 2004). TTR is subjected to vortex induced vibrations which is one of the main reasons behind fatigue design (Hartlen and Currie, 1970; Jauvtis and Williamson, 2004; Liao, 2001; Low and Cheung, 2012; Mukundan et al., 2009). Many researchers (Low and Cheung, 2012; Mukundan et al., 2009) did extensive study on TTR's VIV (Vortex Induced Vibration). In actual sea conditions tether is subjected to parametric excitation because of fluctuation in axial tension caused by heave motion of platform under wave loading and complicated interaction between tether and correlated alternating sequence of vortices (Yang and Xiao, 2014). Parametric excitation causes significant loading when frequency of external excitation and natural frequency of tether matches particular condition (Chandrasekaran et al., 2006; Chatjigeorgiou and Mavrakos, 2005). Many research scholars studied non-linear dynamics of TTR and pointed out because of non-linearity produced by quadratic damping and parametric resonance, wide range of instability is present in TTR system. Due to these non-linear oscillations, there is a huge load coming on the tether caused by environmental fluid forces. This may significantly affect the various operations of structural system and may finally cause catastrophic fatigue failure. In design of TTR it is one of challenging tasks to avoid these instabilities during design of these kinds of offshore systems.

Generally researches only consider VIV condition for dynamic analysis of tethers. For safe design of tether, it is compulsory to consider combined vortex ortex and parametric loading. To obtain the coupled dynamic response of tether, parametric excitation caused by vertical wave forces and variable buoyancy should be considered together with this if amplitude of tension fluctuating is significant, it should be taken into account while obtaining stiffness in surge and sway direction, otherwise it may cause serious dynamic stability problems (Banik and Datta, 2009). Reduction in tether pretension in order to increase the payload capacity of TLP may significantly alter dynamic response and may produce instability (Yang et al., 2009). Parametric instability is also significant in irregular waves, many modern ships experienced serious accident due to parametric roll. The overall aim of present work is to obtain comparative parametric study of TTR and to check effect of these parameters on nonlinear dynamic response and stability

Corresponding Author: Ranjeet Kr. Verma, Department of Naval Architecture and Offshore Engineering, AMET University, Chennai, India 
under the influence of VIV and parametric excitation. The present research can be divided in following sections. Mathematic model of TTR considering VIV and parametric excitation. In which multiple term Galerkin's method is employed to reduce the non-linear PDE to non-linear ODE which is further simulated using iterative technique to obtain dynamic response of system. Results and discussion under combined excitation is explored. Conclusion obtained from present study.

\section{MATERIALS AND METHODS}

Mathematical modeling: In present study, tension leg platform in $300 \mathrm{~m}$ water depth is taken for study, water depth is considered to be equal to length of TTR other details of TTR is presented in Table 1. It is also, considered that first modal frequency is the prime contributor in response and parametric excitation is taken as twice of vortex shedding frequency. This condition gives maximum response to TTR system in this study. Following assumptions is made before deriving the equation of motion:

- The material and mechanical properties are assumed uniform, homogeneous and isotropic along the length of tether

- The velocity of current is considered to be uniform along the length of tether

- Cross-flow oscillation of tether is considered and inline oscillation is neglected

In the tension leg platform of TTR, seabed is considered as origin of coordinate system with $\mathrm{z}$ as vertical coordinate. Water depth is considered to be equal to Length (L) of TTR, T(t) is top tension and $\mathrm{v}(\mathrm{z})$ represents lateral displacement. Along the length of tether current is varying it is represented in +ve $\mathrm{x}$ direction. Only the vibrations caused by alternating sequence of vortices are considered in present situation. The tether can be mathematically modeled as

Table 1: Particulars of TTR

\begin{tabular}{ll}
\hline Particulars & Dimensions \\
\hline Length $(\mathrm{L})$ & $300 \mathrm{~m}$ \\
External Diameter $(\mathrm{D})$ & $1.1176 \mathrm{~m}$ \\
Thickness $(\mathrm{T})$ & $0.038 \mathrm{~m}$ \\
Mass per unit length $(\mathrm{M})$ & $1046 \mathrm{~kg} / \mathrm{m}$ \\
Added mass per unit length $(\mathrm{M})$ & $1010 \mathrm{~kg} / \mathrm{m}$ \\
Total mass per unit length $(\overline{\mathrm{m}})$ & $2056 \mathrm{~kg} / \mathrm{m}$ \\
Bending stiffness $(\mathrm{EI})$ & $3.89 \times 10^{9} \mathrm{~N} / \mathrm{m}^{2}$ \\
Tension ratio $\left(\varepsilon^{\prime}=\mathrm{T} / \mathrm{T}_{0}\right)$ & 0.328 \\
Pretension $\left(\mathrm{T}_{0}\right)$ & $1366 \mathrm{tf}$ \\
Structural damping coefficient $\left(\mathrm{C}^{\prime}\right)$ & $0.319 \times 10^{-3}$ \\
Natural frequency $(\lambda)$ & $0.856 \mathrm{rad} / \mathrm{sec}$ \\
Amplitude of excitation force $(\mathrm{g})$ & $0.126 \mathrm{C}_{\mathrm{L}}$ \\
\hline
\end{tabular}

pinned-pinned end condition beam subjected to pretension T. Due to wave motion, there is tension fluctuation in tether which can be represented by $\mathrm{T} \cos$.t where is frequency of heave motion.

Motion of tether . $(\mathrm{z}, \mathrm{t})$ causes pressure distribution, resulting in drag force and inertial force. Since, D/L. 0.2 for tether, this force can be represented by Morison's equation. In which drag force is proportional to square of velocity $\dot{v}(\mathrm{z}, \mathrm{t})$ and inertial force is proportional to acceleration $\mathfrak{v}(\mathrm{z}, \mathrm{t})$ :

$$
\begin{aligned}
& F_{v}(z, t)=0.5 C_{L} \rho D V_{c}^{2}(z) \cos \lambda t- \\
& C_{a} \rho \pi D^{2} /{ }_{4} \ddot{v}-0.5 C_{D} \rho D \dot{v}|\dot{v}|
\end{aligned}
$$

Dynamic equation of motion of tether considering tension variation and vortex loading can be represented using following equations (Dong et al., 1992):

$$
\begin{aligned}
& \mathrm{EI} \frac{\partial^{4} v}{\partial \mathrm{z}^{4}}-\left(\mathrm{T}_{0}+\mathrm{T} \cos \omega \mathrm{t}\right) \frac{\partial^{2} v}{\partial \mathrm{z}^{2}}+ \\
& \mathrm{C}_{1} \dot{v}+\mathrm{m} \ddot{v}=\mathrm{F}_{\mathrm{y}}(\mathrm{z}, \mathrm{t})
\end{aligned}
$$

Where:

$$
\begin{aligned}
& \text { EI } \quad=\text { Flexural rigidity } \\
& \text { T. }=\text { Pretension } \\
& \mathrm{T}=\text { Amplitude of Tension fluctuation } \\
& \mathrm{m}=\text { Mass of tether per unit length } \\
& \text { C. }=\text { Structural damping coefficient } \\
& \text {. } \quad=\text { Frequency of parametric excitation } \\
& \mathrm{F} .(\mathrm{z}, \mathrm{t})=\text { Total Fluid force on tether per unit length }
\end{aligned}
$$

Tether can be mathematically modeled as pinned-pinned beam, so, deflected shape of tether can be assumed to be a function which satisfies boundary conditions pertaining to pinned-pinned condition:

$$
\begin{aligned}
& v(0, t)=v(1, t)=0 \\
& \left(d^{2} v / d z^{2}\right)_{z=0,1}=0
\end{aligned}
$$

where 1 is the length of tether. Solution of Eq. 1 can be obtained by mode superposition principle. Assuming tether first modal frequency matches with frequency of vortex shedding and neglecting contribution of higher modes in finding response of tether. The error in displacement response obtained by this procedure is very small. But error in SFD and BMD can be around 10-12\%. (Dong and Lou, 1992). With above mentioned conditions response of TTR can be obtained using:

$$
v(\mathrm{z}, \mathrm{t})=v(\mathrm{t}) \sin \frac{\pi \mathrm{z}}{1}
$$


where. $(z, t)$, represents response of tether, Eq. 2 can be simplified with above mentioned assumptions and following equations is obtained:

$$
\begin{gathered}
+\frac{\mathrm{C}_{1}^{\prime}}{\mathrm{m}} \dot{v}+\left[\omega_{\mathrm{b}}^{2}+\omega_{\mathrm{c}}^{2}\left(1+\varepsilon^{\prime} \cos \omega \mathrm{t}\right)\right] \nu+ \\
8 / 3_{\pi \mathrm{m}} \mathrm{K}_{2} \mathrm{C}_{\mathrm{b}} \dot{v}|\dot{v}|=2 \frac{\mathrm{K}_{2} \mathrm{C}_{\mathrm{L}} \cos \lambda \mathrm{t}}{\mathrm{lm}} \int_{0}^{1} \mathrm{~V}_{\mathrm{c}}^{2}(\mathrm{z}) \sin \frac{\pi \mathrm{z}_{\mathrm{m}}}{1} \mathrm{~K}_{2}= \\
0.5 \rho \mathrm{D}, \omega_{\mathrm{b}}^{2}=\left(\frac{\pi}{1}\right)^{4} \frac{\mathrm{EI}}{\mathrm{m}}, \omega_{\mathrm{b}}^{2}=\left(\frac{\pi}{1}\right)^{2} \frac{\mathrm{T}_{0}}{\mathrm{~m}}, \varepsilon^{\prime}=\frac{\mathrm{T}}{\mathrm{T}_{0}} \\
\overline{\mathrm{m}}=\mathrm{m}+\mathrm{C}_{\mathrm{a}} \rho \pi \mathrm{D}^{2} / 4
\end{gathered}
$$

is virtual mass per unit length of tether. Natural frequency because of flexural stiffness is very small as compared to lateral frequency : because of fluctuations in axial tension. Maximum tether response occurs when frequency of parametric excitation is twice the vortex shedding frequency (Dong and Lou, 1992). Introducing book keeping parameters and keeping other parameters constant, neglecting $\ldots$ and simplifying right hand side of Eq. 5, following equations can be obtained:

$$
\begin{aligned}
& \omega_{\mathrm{c}}^{2}=\lambda^{2}+\gamma^{\prime} \\
& \ddot{\mathrm{y}}+v \mathrm{C}_{1} \dot{v}+v \mathrm{C}_{2} v^{2} \operatorname{sign}(\dot{v})+\left(\lambda^{2}+v \gamma+v \varepsilon \lambda^{2} \cos 2 \lambda \mathrm{t}\right) v=(7) \\
& v g \cos \lambda \mathrm{t}
\end{aligned}
$$

where is small parameter:

$$
\begin{aligned}
& \mathrm{c}_{1}=\mathrm{C}_{1}^{\prime} / \mathrm{vm}^{\prime} \mathrm{c}_{2}=8 \mathrm{~K}_{2} \mathrm{C}_{\mathrm{D}} / 3 \pi \overline{\mathrm{m}} \mathrm{v}, \varepsilon=\varepsilon^{\prime} / \mathrm{vg}= \\
& \frac{2 \mathrm{~K}_{2} \mathrm{C}_{\mathrm{L}}}{\operatorname{lm} \mathrm{m}} \int_{0}^{1} \mathrm{~V}_{\mathrm{c}}^{2}(\mathrm{z}) \sin \frac{\pi \mathrm{z}}{1} \mathrm{dz} \\
& \gamma=\gamma / v, \operatorname{sign}(\dot{v})
\end{aligned}
$$

is written to preserve sign of $\dot{y}$ and higher order : has been neglected.

\section{RESULTS AND DISCUSSION}

Non-linear dynamic response and stability analysis of TTR is obtained considering a single degree of freedom system using iterative technique.

Effect of pretension: The 2 and $3 \mathrm{D}$ phase plot shown in Fig. $1 \mathrm{a}, \mathrm{b}$ is for the given value of $\mathrm{C}=0.1, \mathrm{C}=0.6 \mathrm{v}_{\mathrm{v}}=0, \mathrm{~s}=$ $0.328, y=0, d y=0$. It is observed that after few cycles of dynamic response, system attains constant amplitude of $7.5 \mathrm{~m}$ and enters into stable limit cycle.
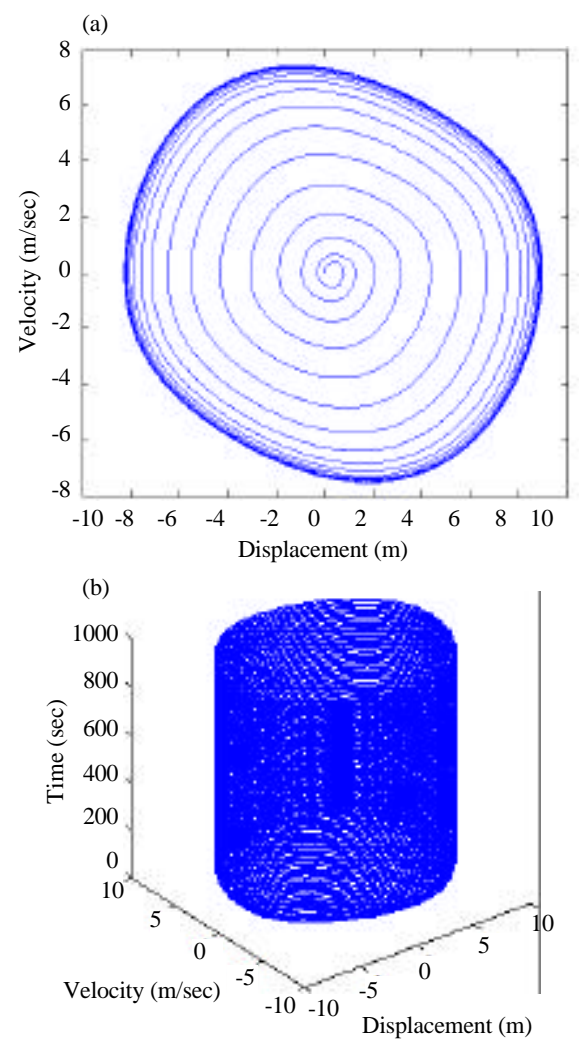

Fig. 1: a) Phase potrait; C. $=0.1, \mathrm{C}=0.6,{ }_{v}=0, \mathrm{~s}=0.328, \mathrm{y}$ $=0, \mathrm{dy}=0$ and $\mathrm{b}) 3 \mathrm{D}$ phase potrait; $\mathrm{C}=0.1, \mathrm{C}$. $=0.6, v=0,:=0.328, y=0, d y=0$

The effect of reducing the pretension value by $20 \%$ of initial value is shown in Fig. $2 \mathrm{a}, \mathrm{b}$ for the given values of $\mathrm{C} .=0.1, \mathrm{C}=0.6, \mathrm{r}=0.2856,=0.328, \mathrm{y}=0 \mathrm{dy}=0$, it is observed that reducing the pretension value raises the stability issues for the tethers which is evident by phase portrait shown in Fig. 2a several ellipses starts crossing each other and beating phenomenon, although, beating phenomenon is not pronounced after $600 \mathrm{sec}$.

The effect of reducing the pretension value by $40 \%$ of initial value is shown in Fig. $3 \mathrm{a}, \mathrm{b}$ for the given values of it is observed that it further enhances the instability issues in tether, although, amplitude of response decreases as compared to Fig. 2a, b showing very dense overlapping ellipses in 2 and $3 \mathrm{D}$ phase portrait which is crossing each other.

Effect of drag coefficient and lift coefficient: Comparing Fig. $4 \mathrm{a}, \mathrm{b}$ and $5 \mathrm{a}, \mathrm{b}$ for the given values $\mathrm{C}=0.3, \mathrm{C}=0.6, \mathrm{v}$ $=0.1429, \mathrm{~s}=0.328, \mathrm{y}=0 \mathrm{dy}=0$ and $\mathrm{C}=0.6, \mathrm{C}_{\mathrm{s}}=0.6 \mathrm{,}_{\mathrm{v}}=$ $0.1429,=0.328, y=0 \mathrm{dy}=0$, respectively, it is observed that by increasing the value of drag coefficient, causes reduction in response amplitude but increases the stability issues for tether. 

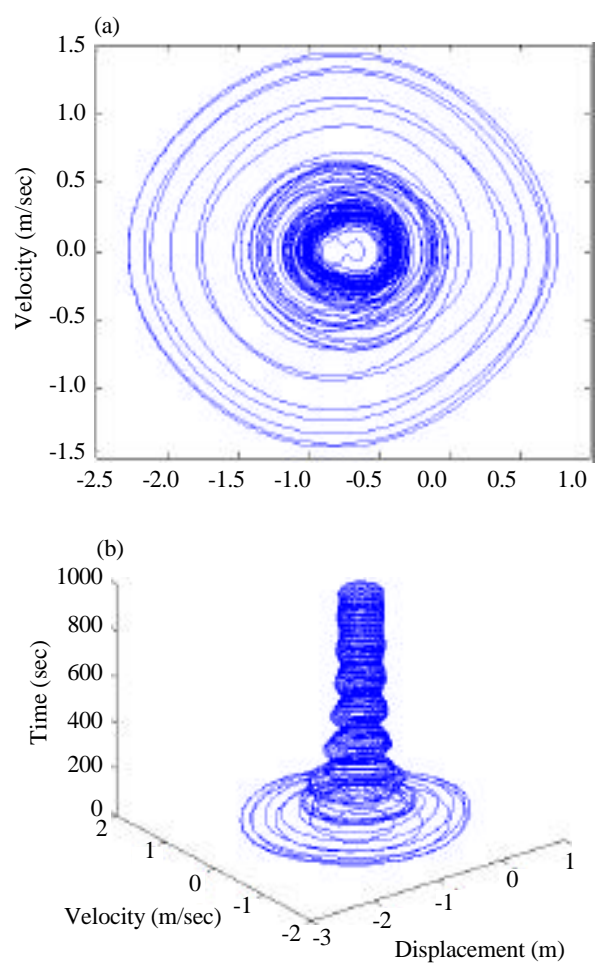

Fig. 2: a) Phase potrait; $C=0.1, C=0.6, v=0.1429, y=0$, $\mathrm{dy}=0$ and b) 3D phase plot

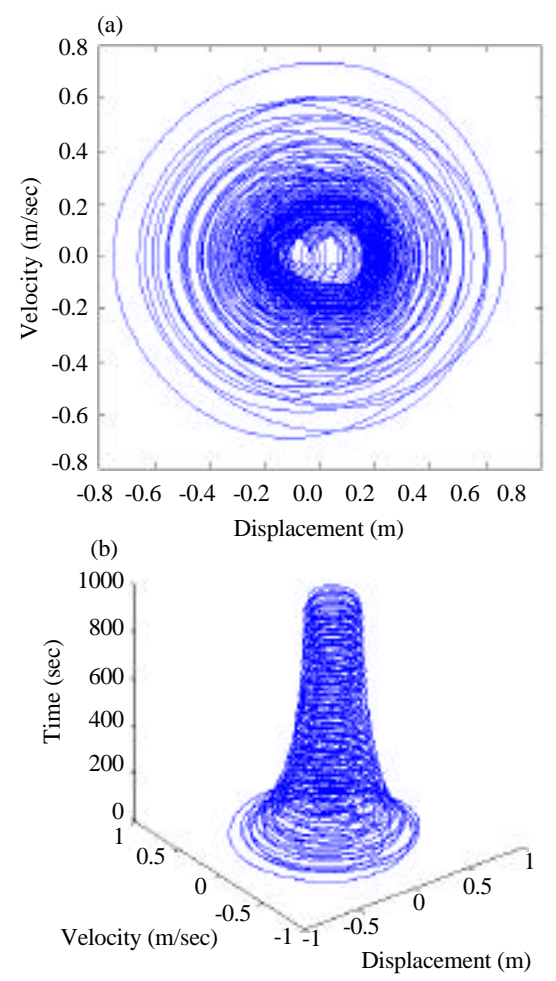

Fig. 3: a) Phase potrait; $C=0.1, C=0.6, r=0.2856, y=0$, $\mathrm{dy}=0$ and b) $3 \mathrm{D}$ phase plot; $\mathrm{C} .=0.1,{ }_{\mathrm{a}}=0.6$, $\mathrm{v}=0.2666, \mathrm{y}=0, \mathrm{dy}=0$
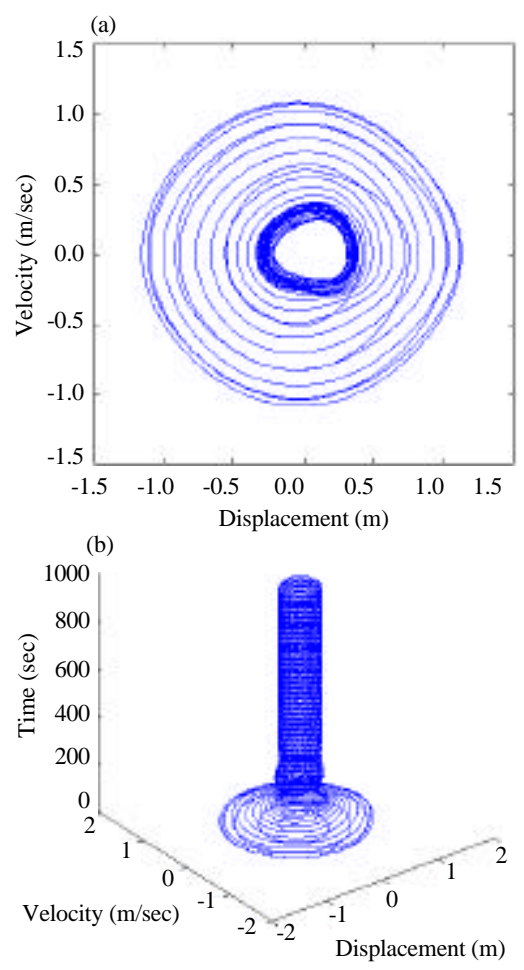

Fig. 4: a) Phase potrait; $C=0.1, C=0.6, v=0.1429, y=0$, $\mathrm{dy}=0$ and $\mathrm{b}) 3 \mathrm{D}$ phase plot; $\mathrm{C} .=0.1, \mathrm{C}=0.6$, $r=0.1429, y=0, d y=0$
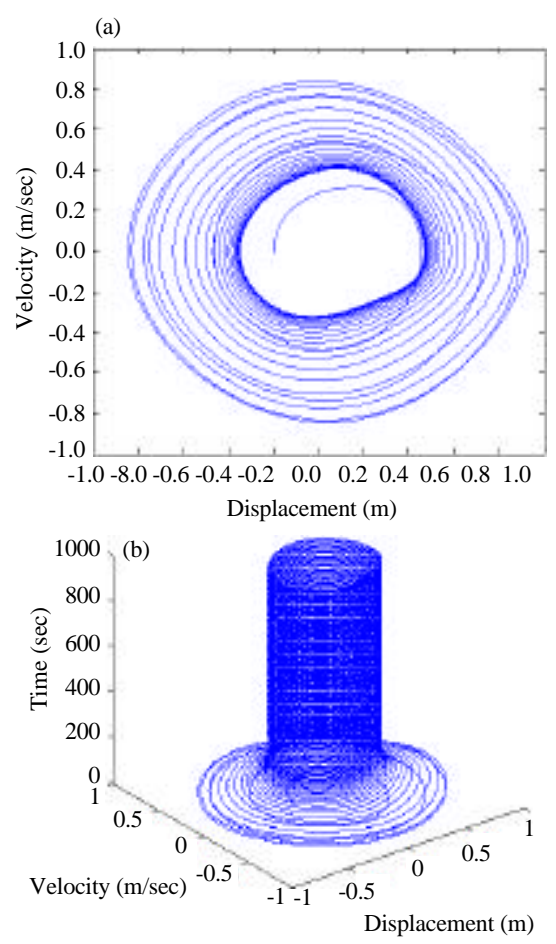

Fig. 5: a) Phase potrait; $C=0.6, C=0.6, r=0.1429, y=0$, $\mathrm{dy}=0$ and b) $3 \mathrm{D}$ phase plot; $\mathrm{C}=0.6, \mathrm{C}=0.6$, $r=0.1429, y=0, d y=0$ 

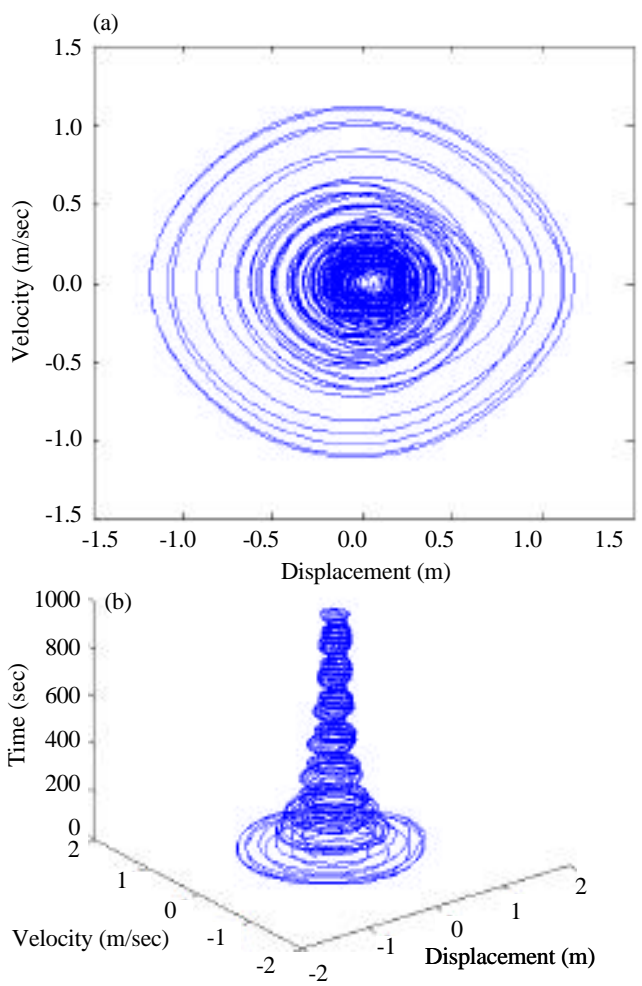

Fig. 6: a) Phase potrait; $\mathrm{C}=0.1, \mathrm{C}=0.3, \mathrm{v}=0.1429_{2}=$ $0.328, \mathrm{y}=0, \mathrm{dy}=0$ and $\mathrm{b}$ ) $3 \mathrm{D}$ phase plot; $\mathrm{C}=0.1$, $\mathrm{C}=0.3{ }_{r}=0.1429, \mathrm{~s}=0.328, \mathrm{y}=0, \mathrm{dy}=0$
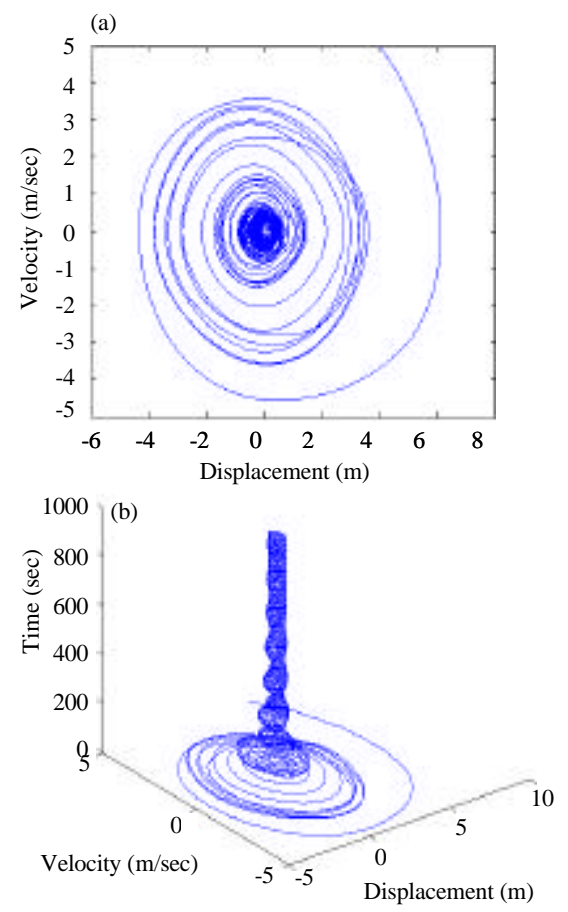

Fig. 7: a) Phase plot; $C=0.1, C=0.6, r=0.1429, y=5$, dy $=5$ and b) $3 \mathrm{D}$ phase plot and b) $\mathrm{C}=0.1, \mathrm{C}=0.6{ }_{r}=$ $0.1429, y=5, d y=5$

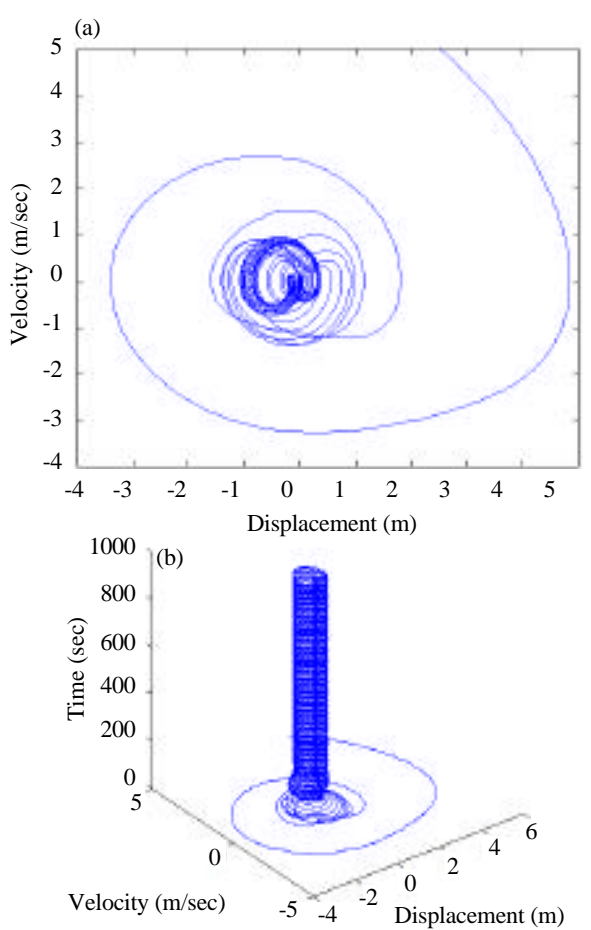

Fig. 8: a) Phase plot; C. $=0.3, C=0.6, r=0.1429, y=5$, dy $=5$ and b) $3 \mathrm{D}$ phase plot; $\mathrm{C} .=0.3,{ }_{a}=0.6,{ }_{\mathrm{v}}=$ $0.1429, y=5, d y=5$

Comparing Fig. $6 \mathrm{a}, \mathrm{b}$ and $2 \mathrm{a}, \mathrm{b}$ it is observed that change in lift coefficient from, $\mathrm{C}_{\mathrm{s}}=0.3$, to higher value $\mathrm{C}=0.6$, slightly affects the response of tether.

Effect of initial conditions: Comparing the Fig. 2a, b and $7 \mathrm{a}, \mathrm{b}$, it is observed that by changing the initial conditions $\mathrm{y}=0, \mathrm{dy}=0$ to higher values $\mathrm{y}=5, \mathrm{dy}=5$ doesn't affect the pattern of response significantly but amplitude is increased slightly for higher value of I.C but after $300 \mathrm{~s}$ becomes approximately same.

Comparing the Fig. $4 \mathrm{a}, \mathrm{b}$ and $8 \mathrm{a}, \mathrm{b}$, it is observed that by changing the initial conditions, $y=0, d y=0$ to higher values $\mathrm{y}=5, \mathrm{dy}=5$ instability in system increase which is evident in phase portrait (Fig. 8b) various ellipse are overlapping on each other.

\section{CONCLUSION}

In present study dynamic analysis of TTR is simulated considering vortex induced vibration and parametric excitation and various design parameters of TTR is evaluated for safe design. Based upon the work presented in this paper following concluding remarks can be made. Decrease in pretension value increases the response amplitude and instability in the tether system 
because of multiplicative load present in the system. Increase in drag coefficient value decreases the amplitude of response but at higher value of initial conditions cause's significant instability in the system. This is because of drag coefficient contribution to multiplicative loading. Altering the lift coefficient slightly affects the response of tether under above mentioned conditions. This is because of lift coefficient contribution to additive loading.

\section{REFERENCES}

Banik, A.K. and T.K. Datta, 2009. Stability analysis of TLP tethers under vortex-induced oscillations. J. Offshore Mech. Arct. Eng., 131: 1-7.

Chandrasekaran, S., N.R. Chandak and G. Anupam, 2006. Stability analysis of TLP tethers. Ocean Eng., 33: 471-482.

Chatjigeorgiou, I.K. and S.A. Mavrakos, 2005. Nonlinear resonances of parametrically excited risers-numerical and analytic investigation for $\mathrm{U}=2 \mathrm{u} 1$. Comput. Struct., 83: 560-573.

Chen, W., M. Li, Z. Zheng and T. Tan, 2012. Dynamic characteristics and VIV of deepwater riser with axially varying structural properties. Ocean Eng., 42: $7-12$.

Dong, Y. and J.Y. Lou, 1992. A study on stability of tension leg platform nonlinear oscillation induced by vortex shedding. National Academies of Sciences, Engineering and Medicine, Washington, DC., USA. https://trid.trb.org/view.aspx?id=442311

Hartlen, R.T. and I.G. Currie, 1970. Lift-oscillator model of vortex-induced vibration. J. Eng. Mech. Div., 96: $577-591$.
Jauvtis, N. and C.H.K. Williamson, 2004. The effect of two degrees of freedom on vortex-induced vibration at low mass and damping. J. Fluid Mech., 509: 23-62.

Josefsson, P.M. and C. Dalton, 2010. An analytical/computational approach in assessing vortex-induced vibration of a variable tension riser. J. Offshore Mech. Arct. Eng., 132: 1-7.

Liao, J.C., 2001. Vortex-induced vibration of slender structures in unsteady flow. Ph.D Thesis, Massachusetts Institute of Technology, Cambridge, Massachusetts.

Low, Y.M. and S.H. Cheung, 2012. On the long-term fatigue assessment of mooring and riser systems. Ocean Eng., 53: 60-71.

Mukundan, H., Y. Modarres-Sadeghi, J.M. Dahl, F.S. Hover and M.S. Triantafyllou, 2009. Monitoring VIV fatigue damage on marine risers. J. Fluids Struct., 25: 617-628.

Sanaati, B. and N. Kato, 2012. A study on the effects of axial stiffness and pre-tension on VIV dynamics of a flexible cylinder in uniform cross-flow. Appl. Ocean Res., 37: 198-210.

Wilde, J.J.D. and R.H. Huijsmans, 2004. Laboratory investigation of long riser VIV response. Proceedings of the 14th International Conference on Offshore and Polar Engineering, May 23-28, 2004, ISOPE, Toulon, France, pp: 1-6.

Yang and Zhen and L. Huajun, 2009. Instability assessment of deep-sea risers under parametric excitation. China Ocean Eng. English, 23: 603-612.

Yang, H. and F. Xiao, 2014. Instability analyses of a top-tensioned riser under combined vortex and multi-frequency parametric excitations. Ocean Eng., 81: $12-28$. 